\title{
Blood flow simulation in the aorta with aortic valves using the regularized lattice Boltzmann method with LES model
}

\author{
T. Fukui \& K. Morinishi \\ Kyoto Institute of Technology, Japan
}

\begin{abstract}
The heart pumps blood throughout the body efficiently owing to the heart valves operation. The sinus of Valsalva, which is located on the beginning of aorta, is considered to affect the aortic valve movements. When blood ejects from the heart, vortices are observed within the sinus of Valsalva. This vortical motion has the advantage of preventing the valve leaflet from bulging outward to contact the walls of the sinuses. Moreover, since the sinus of Valsalva lies at the beginning of coronary arteries, vortices within the sinus could affect the flow rate into the coronary arteries. It is therefore important to investigate the vortices within the sinus of Valsalva quantitatively to clarify the mechanism of aortic valve movements. In this study, we apply the virtual flux method (VFM), which is a tool to describe stationary or moving body shapes, to the $2 \mathrm{D}$ aortic valves and reproduce the blood flow fields with high Reynolds number around the aortic valves by regularized lattice Boltzmann method with LES model, and estimate the WSS distribution on the valve and sinus wall to consider the atherosclerosis generation. As a result, we found that the WSS on the LV-facing surface is larger than that on the aortic-facing surface, and the WSS on the distal end wall of the sinus of Valsalva is higher due to vortices generation. The vortices' distribution in time and space is important to consider both in the mechanism of aortic valve movements and the generation of cardiovascular disease such as aortic stenosis.

Keywords: aortic valves, blood flow simulation, regularized lattice Boltzmann method, LES model, sinus of Valsalva, wall shear stress, atherosclerosis, vorticity.
\end{abstract}




\section{Introduction}

The aortic valve consists of three thin membranous cusps, which in the open position are displaced outward toward the aorta to eject blood in the left ventricle, and come together to seal the aortic orifice in the closed position [1]. The heart pumps blood throughout the body efficiently owing to the heart valves operation.

The sinus of Valsalva, which is located on the beginning of aorta, is considered to affect the aortic valve movements. When blood ejects from the heart, vortices are observed within the sinus of Valsalva. This vortical motion has the advantage of preventing the valve leaflet from bulging outward to contact the walls of the sinuses. The open sinus chamber thus can be supplied with fluid to fill the increasing volume behind the valve leaflets as they move toward closure. Bellhouse and Talbot [2] suggested that the trapped vortex within the sinus also interacts with the decelerating flow field and thus pushes the leaflets into the aorta. To study the role of the sinus of Valsalva in valve closure at the physiological value of the Strouhal number, van Steenhoven and van Dongen [3] assessed the influence of the shape of the sinus on valve closure. They showed the presence of a cavity of a certain minimum size is essential in both axial and radial directions for the mechanism of valve closure in the deceleration phase of systole. A larger ratio of sinus radius to leaflet length than the physiological one appears to result in a faster closure. It is therefore important to investigate the vortices within the sinus of Valsalva quantitatively to clarify the mechanism of aortic valve movements. Moreover, since the sinus of Valsalva lies on the beginning of coronary arteries, vortices within the sinus could affect the flow rate to the coronary arteries. Vortices developed from the aortic valve leaflets are also important for the coronary circulation.

In the past study, we performed numerical simulation of blood flows around aortic valves by lattice Boltzmann method [4, 5]. The method of lattice Boltzmann equation (LBM) is a simple kinetic-based approach for fluid flow computation. The LBM has advantages its simple coding and its locality, which makes it intrinsically parallelizable [6], and has been applied to many general problems [7-9], and those relevant to blood flow simulation [10-12], as well. However, stability of the simulation by LBM is strictly dependent on its relaxation time, which leads to restriction of the Reynolds number. Since blood flow simulation in the aorta is accompanied by high Reynolds number, it is necessary to improve stability of LBE. Izham et al. [13] proposed regularized lattice Boltzmann method (RLBM), which is based on the observation of symmetric condition in Chapman-Enskog expansion by Latt and Chopard [14], and it has successfully achieved higher stability in numerical simulation at high Reynolds number. In this study, we apply the virtual flux method (VFM) [15], which is a tool to describe stationary or moving body shapes in a Cartesian grid, to the 2D aortic valves and reproduce the blood flow fields around the aortic valves and the sinus of Valsalva by RLBM with LES model, and estimate the WSS distribution on the valve and sinus wall to consider the atherosclerosis generation. 


\section{Methods}

\subsection{Computational model}

Numerical simulation of blood flow in aorta with valves and sinus of Valsalva is performed. Figure 1 shows the schematic view of the 2-dimensional axisymmetric simulation model used in this study. The longitudinal length $L$ and diameter $D$ are set to $200 \mathrm{~mm}$ and $20 \mathrm{~mm}$. The valve leaflets are modeled as arcs of a circle with a radius of $D / \sqrt{2}$, and placed at $80 \mathrm{~mm}(4 D)$ from the inlet. The shape of the sinus of Valsalva is approximated as a semi-ellipse. The longitudinal length $a$ and depth $b$ of the sinus of Valsalva are set to $30 \mathrm{~mm}$ and $15 \mathrm{~mm}$, respectively. The arterial wall and valves are described by virtual flux method on Cartesian coordinate as described below.

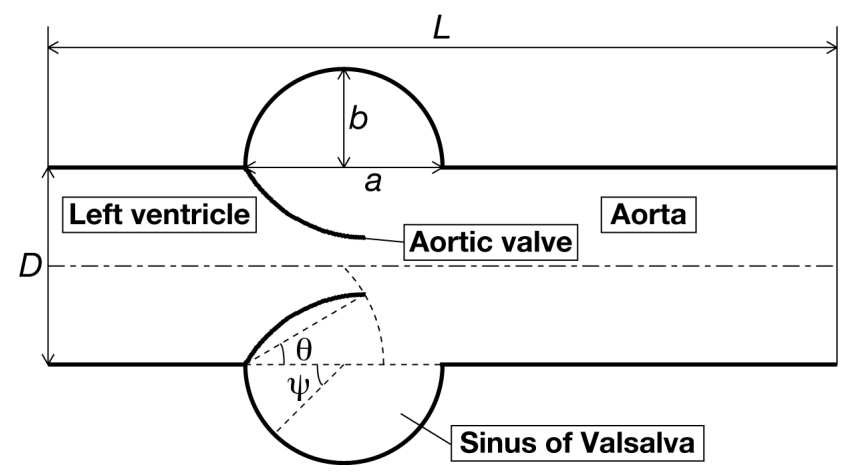

Figure 1: $\quad$ Schematic view of the computational model.

\subsection{Governing equations}

\subsubsection{Lattice Boltzmann method}

The discrete velocity Boltzmann equation (DVBE) is as follows,

$$
\frac{\partial f_{\alpha}}{\partial t}+\boldsymbol{e}_{\alpha} \cdot \nabla f_{\alpha}=\Omega_{\alpha},
$$

where $\boldsymbol{e}_{\zeta}$ is the discrete particle velocity, $f_{<}$is the distribution function associated with $\boldsymbol{e}_{\beta}$, and $\wedge_{\gamma}$ is the collision operator. The collision operator, which is very complicated, is usually approximated by the simple single-relaxation-time Bhatnagar-Gross-Krook (BGK) model [16]:

$$
\Omega_{\alpha}=-\frac{1}{\tau}\left(f_{\alpha}-f_{\alpha}^{(e q)}\right),
$$

where $f_{\zeta}^{(e q)}$ is the equilibrium distribution function, and / is the relaxation time. The evolution of the distribution function $f_{<}$for the lattice Boltzmann equation can be written as

$$
f_{\alpha}\left(\boldsymbol{x}+\boldsymbol{e}_{\alpha} \Delta t, t+\Delta t\right)-f_{\alpha}(\boldsymbol{x}, t)=-\frac{1}{\tau}\left\{f_{\alpha}(\boldsymbol{x}, t)-f_{\alpha}^{(e q)}(\boldsymbol{x}, t)\right\},
$$


In this study, we use a 2D square lattice model with 9 velocities, which is referred to as the D2Q9 model.

It is shown that the Navier-Stokes equations can be derived from the LBM though a Chapman-Enskog expansion procedure in the incompressible limit [17] with a relaxation time / as

$$
\tau=\frac{3 v}{c \delta x}+\frac{\delta t}{2}
$$

The most common choice for the equilibrium distribution function $f_{\zeta}^{(e q)}$ is the truncated form of the Maxwell distribution, which is a very good approximation for small Mach numbers [18].

$$
f_{\alpha}^{(e q)}=\omega_{\alpha} \propto\left[1+\frac{3\left(\boldsymbol{e}_{\alpha} \cdot \boldsymbol{u}\right)}{c^{2}}+\frac{9\left(\boldsymbol{e}_{\alpha} \cdot \boldsymbol{u}\right)^{2}}{2 c^{4}}-\frac{3 \boldsymbol{u}^{2}}{2 c^{2}}\right\rfloor,
$$

where $7_{\text {is }}$ is the weight coefficients.

\subsubsection{Regularized lattice Boltzmann method}

The single-relaxation-time (SRT) LBM has been widely used for its simplicity, efficiency and ease of parallel programming implementation, however, it requires relatively large number of grids to simulate flows at even moderately high Reynolds number. In Chapman-Enskog expansion procedure, the nonequilibrium part of first-order is symmetrical with respect to spatial reflection. Latt and Chopard [14] observed that this symmetric condition is not necessarily satisfied and appear to take a non-negligible value in numerical simulations using SRT-LBM. Based on this observation, they considered a regularization step that enforces symmetrical property and proposed regularized lattice Boltzmann method (RLBM).

The relationships between stress tensor and distribution function in RLBM [13] is defined as

$$
\Pi_{i j}=\sum_{\alpha} e_{\alpha i} e_{\alpha j} f_{\alpha},
$$

where $\angle_{i j}$ is the stress tensor. The non-equilibrium part of the distribution and stress tensor were given as

$$
\begin{gathered}
f_{\alpha}^{(\text {neq })}=f_{\alpha}-f_{\alpha}^{(e q)}, \\
\Pi_{i j}^{(n e q)}=\Pi_{i j}-\Pi_{i j}^{(e q)} .
\end{gathered}
$$

From the Chapman-Enskog expansion, the non-equilibrium part of $\sum$-order can be explicitly derived as below,

$$
\begin{gathered}
f_{\alpha}^{(\text {neq })} \approx f_{\alpha}^{1}=-\frac{\delta t}{c_{s}^{2}} \tau \omega_{\alpha} \mathbf{Q}_{\alpha i j} \partial_{i} \rho u_{j}, \\
\Pi_{i j}^{(\text {neq })} \approx \sum_{\alpha} e_{\alpha i} e_{\alpha j} f_{\alpha}^{1}=-\delta t c_{s}^{2} \tau\left(\partial_{i} \rho u_{j}+\partial_{j} \rho u_{i}\right),
\end{gathered}
$$

where $\mathbf{Q}_{i j}$ is defined as

$$
\mathbf{Q}_{\alpha i j}=e_{\alpha i} e_{\alpha j}-c_{s}^{2} \delta_{i j},
$$

where $c_{S}$ is the sound speed, and $f_{\zeta}^{1}$ is then written as 


$$
f_{\alpha}^{1}=\frac{\omega_{\alpha}}{2 c_{s}^{4}} \mathbf{Q}_{\alpha i j} \Pi_{i j}^{(n e q)}
$$

By enforcing $f_{\zeta}^{(\text {neq })}=f_{\zeta}^{1}$, the final form of the relaxation process can be written as

$$
f_{\alpha}=f_{\alpha}^{(e q)}+\left(1-\frac{1}{\tau}\right) f_{\alpha}^{1} .
$$

The viscous stress tensor $/{ }_{i j}$ can be evaluated using the non-equilibrium part of the distribution function [19] as

$$
\tau_{i j}=\left(1-\frac{1}{2 \tau}\right) \sum_{\alpha} f_{\alpha}^{(\text {neq })}(x, t)\left(e_{\alpha i} e_{\alpha j}-\frac{1}{D} \boldsymbol{e}_{\alpha} \cdot \boldsymbol{e}_{\alpha} \delta_{i j}\right) .
$$

\subsubsection{Large eddy simulation model}

In the LES model, the relaxation time / is written as

$$
\tau=\tau_{0}+\tau_{t},
$$

where $/_{0}$ and $I_{t}$ are the relaxation times corresponding to molecular viscosity $v_{0}$ and the turbulence or eddy viscosity $v_{t}$ [20]. A quadratic equation for $/ t$ with Smagorinsky constant $C_{s}$ and a filter length scale $\Delta x$ is

$$
\tau_{t}=\frac{1}{2}\left(\sqrt{\tau_{0}^{2}+2\left(C_{s} \Delta x\right)^{2}\left(\rho_{0} c_{s}^{4} \delta t\right)^{-1} \bar{Q}}-\tau_{0}\right),
$$

where $Q$ is the filtered mean momentum flux. In this study, the Smagorinsky constant $C_{s}$ is set to 0.1 as Yu et al. [20] mentioned in the paper above.

\subsubsection{Aortic valves movement}

The aortic valves are assumed to be rigid, and their motion obeys following rigid-body rotation

$$
\boldsymbol{T}=I \frac{d \omega}{d t},
$$

where $\boldsymbol{T}$ is the torque, $I$ is the inertia moment, and 7 is the angular velocity of the valve. The torque $\boldsymbol{T}$ is evaluated by force differences between $\mathrm{LV}$-facing and aortic-facing surfaces of the valve,

$$
\boldsymbol{T}=\sum\left(\boldsymbol{f}^{\text {Ao }}-\boldsymbol{f}^{\mathrm{LV}}\right) \cdot r
$$

where $r$ is the radius of rotation. The forces acting on the valve are obtained by pressure $p$ and viscous stress $/$ ii as follows,

$$
\boldsymbol{f}=\left(p+\tau_{i i}\right) \cdot d r
$$

The inertia moment of the valve $I$ is estimated by assuming the density of the valve is equal to that of the blood. The angular velocity 7 is obtained by firstorder Euler method.

$$
\omega(t+\Delta t)=\omega(t)+\Delta t \frac{T(t)}{I}
$$

\subsection{Boundary conditions}

The virtual flux method (VFM) enables us to estimate flow field around arbitrary body shapes properly in a Cartesian grid [15]. In this study, we apply the VFM to 
express arbitrary body shapes appropriately in case that boundary points are not located on the cell vertex. Figure 2 shows an example of virtual flux boundary, where the virtual boundary point $b$ is placed between cell vertexes 1 and 3 . When the distribution function at vertex 1 is obtained, the distribution function at vertex 3 , which includes the effect of the virtual boundary, is necessary, and vice versa. The macroscopic quantities on the virtual boundary point $b$ are then determined to satisfy the boundary conditions. No-slip condition on the boundary, for example, is attained to assume zero pressure gradient and zero velocity on the boundary.

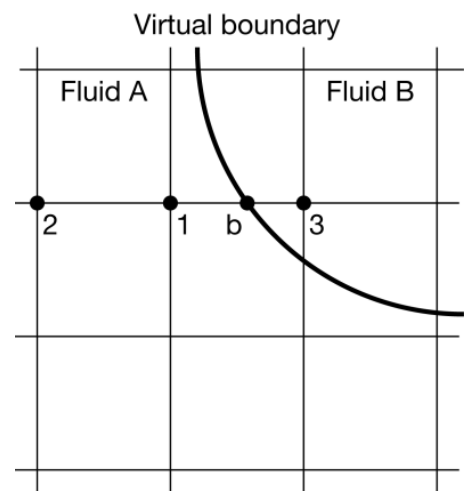

Figure 2: Schematic view of the virtual boundary in a Cartesian grid. The virtual boundary separates Fluid A from Fluid B completely.

Next, the equilibrium distribution function $f_{\zeta}^{(e q)}$ and distribution function $f_{\zeta}$ at the virtual boundary point $b$ are obtained from the macroscopic quantities there. The distribution function $f_{<}$at the vertex 3 is then estimated to extrapolate that at the virtual boundary point $b$.

Axial velocity $u$ at the inlet and pressure $p$ at the outlet are given as shown in Fig. 3, which are modeled as blood flow from left ventricle and aortic pressure,

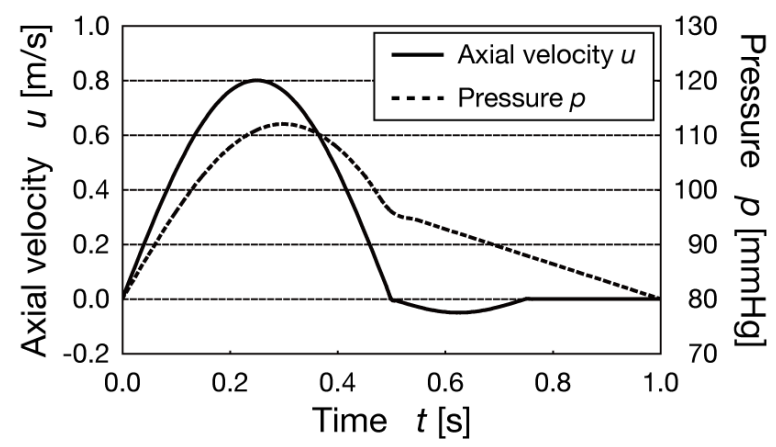

Figure 3: Axial velocity at the inlet and pressure at the outlet. The Reynolds number $R e$ at the peak velocity corresponds to 2,000 . The period of the cardiac cycle is set to 1.0 [s]. 
respectively. Other parameters are linearly extrapolated. No-slip conditions are assumed on the wall and aortic valves. The Reynolds number Re at the peak velocity in Fig. 3 corresponds to 2,000. The period of the cycle is set to 1.0 [s], and totally eight cardiac cycles, including three pre-cycles as preparation, are conducted for the blood flow simulation.

\section{Results and discussion}

Figure 4 shows the vorticity distribution and velocity vectors on the left, and wall shear stress (WSS) diagram on the lower aortic valve on the right in the fourth cardiac cycle at the time interval of 0.1 [s]. The WSS diagram consists of that on the left ventricular (LV)-facing surface and that on the aortic-facing surface, which are denoted by bold and thin lines, respectively. When the pressure at the LV exceeds that on the aorta, the valves start to open and blood flows toward the aorta passing through the aortic orifice. Vortices evolved from the tip of the valve spread and strike on the distal edge of the sinus of Valsalva, then they are divided into multiple. These vortical motions then lead blood flow fields in the aorta to be more complicated. The valves start closing motion and seal the aortic orifice when the blood flow from the LV stops. Since multiple vortices still remain within the sinus of Valsalva in diastole, the aortic valve movements in systole have minor differences from cycle to cycle due to these vortices.

The WSS on the LV-facing surface is remarkably larger than that on the aortic-facing surface in cardiac cycle. It reaches around $40[\mathrm{~Pa}]$ at the center of the valve position in systole owing to vena contracta, which is high physiologically. On the other hand, the WSS distribution on the aortic-facing surface is relatively flat, however, it has a maximal value at the tip of the valve in diastole. The WSS distribution on the aortic valve in time and space differs between LV-facing and aortic-facing surfaces. These findings are important to consider development of atherosclerosis or aortic valve stenosis. No significant differences can be observed between WSS distribution on the upper valve and that on the lower valve.

Figure 5 shows the time-averaged WSS distribution on the wall of sinus of Valsalva for five cardiac cycles. The bold and thin lines represent upper and lower sinus walls, respectively. The sinus position $\psi=0$ and $\pi$ [rad] correspond to proximal and distal edges of the sinus of Valsalva, respectively as shown in Fig. 1. There are no significant differences between WSS distribution on the upper sinus wall and that on the lower sinus wall. The WSS value of the range of $\psi=0$ to $2 \pi / 3$ is almost the same and is around $1.0[\mathrm{~Pa}$. Then it increases to $5.0[\mathrm{~Pa}]$ toward the distal edge of the sinus. This localized high WSS region is mainly attributed to vortices within the sinus of Valsalva as mentioned above. Since the sinus of Valsalva lies on the beginning of coronary arteries, these vortices may affect the flow rate to the coronary arteries as well. Vortices around the aortic valves are therefore important to consider circulation in cardiovascular system as well as WSS distribution. 

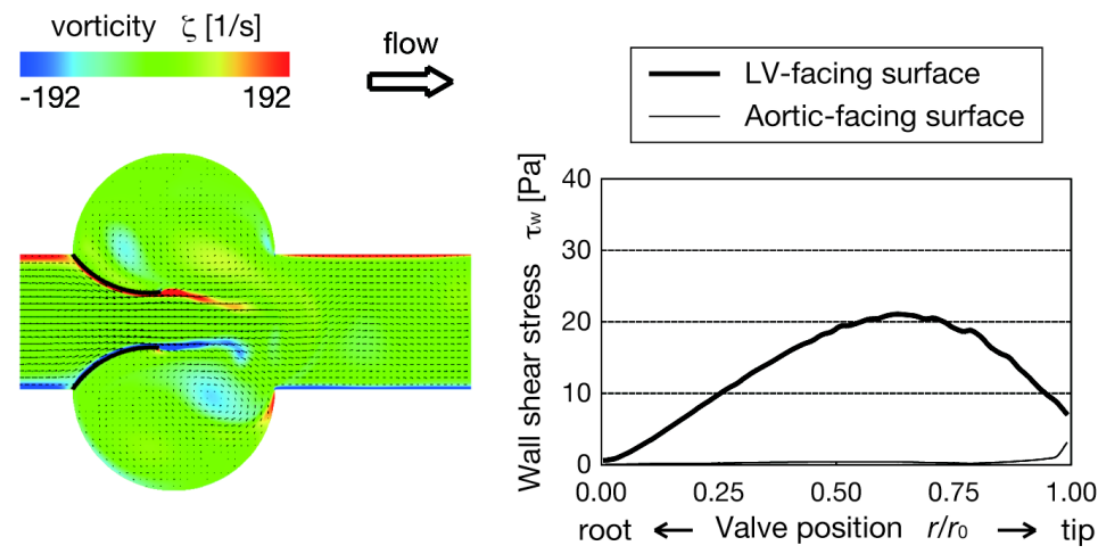

$t=4.1[\mathrm{~s}]$
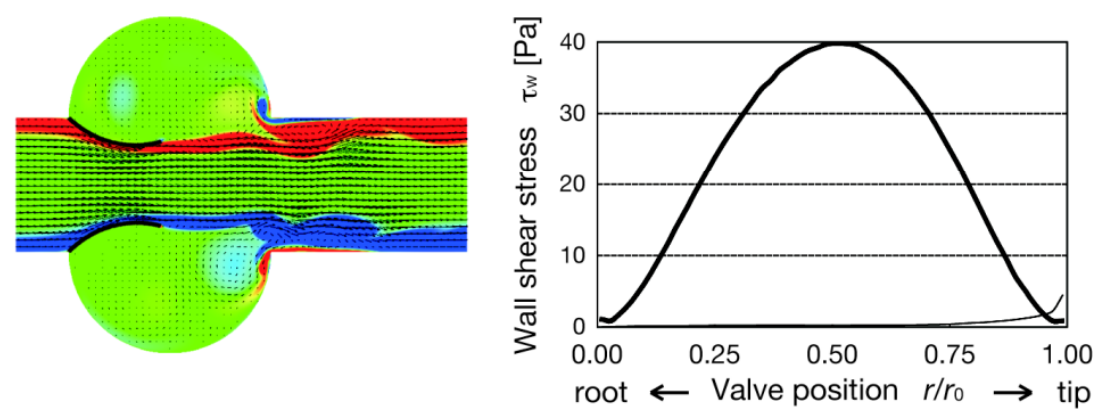

$t=4.2[\mathrm{~s}]$
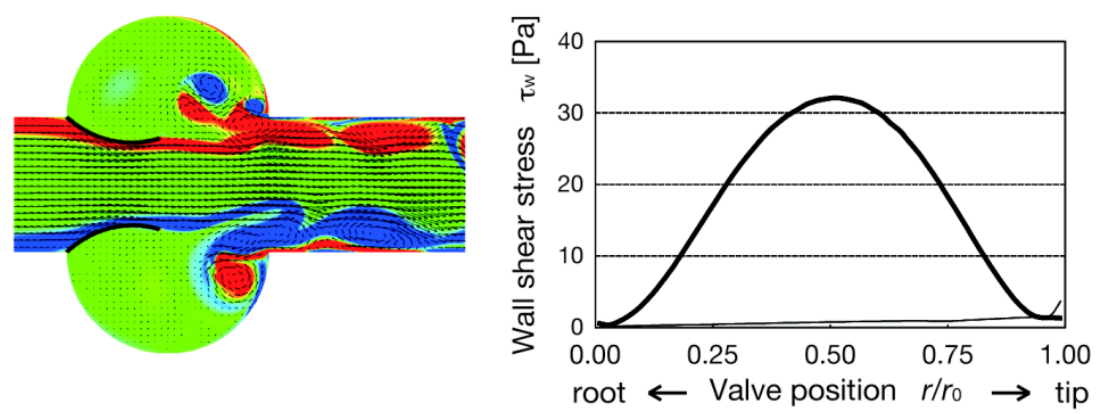

$t=4.3[\mathrm{~s}]$

Figure 4: Vorticity distribution and velocity vectors, and wall shear stress diagram on the lower aortic valve in the fourth cardiac cycle at the time interval of 0.1 [s]. 

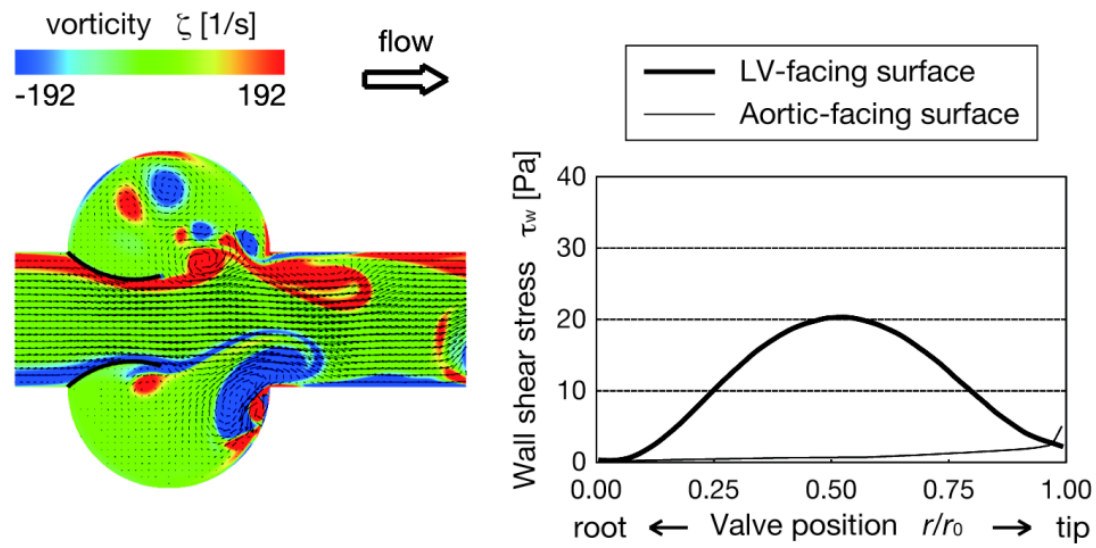

$t=4.4[\mathrm{~s}]$
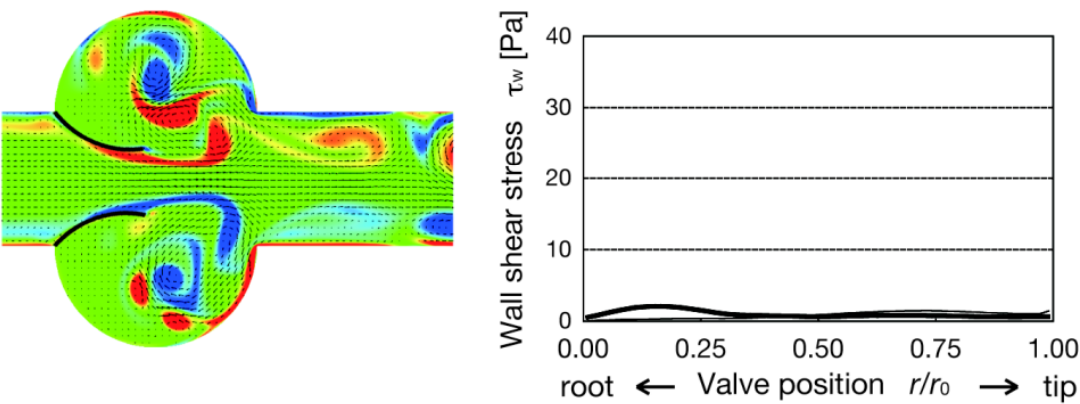

$t=4.5[\mathrm{~s}]$
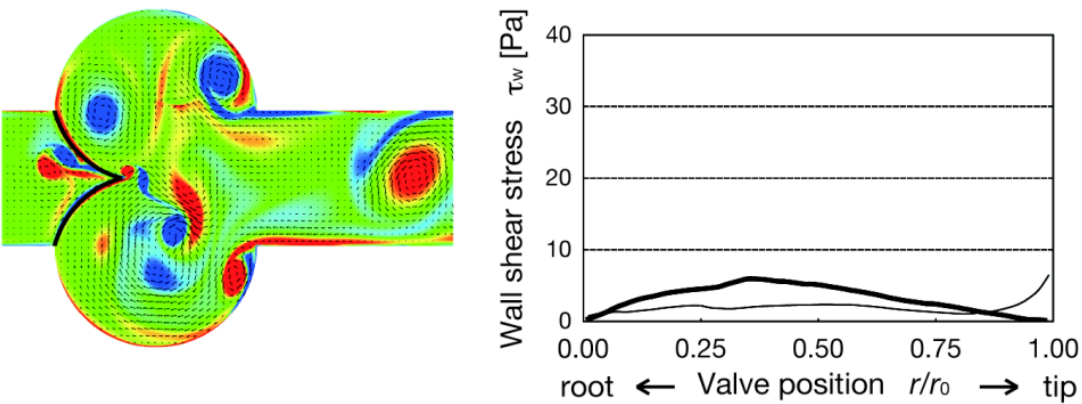

$t=4.6[\mathrm{~s}]$

Figure 4: Continued. 


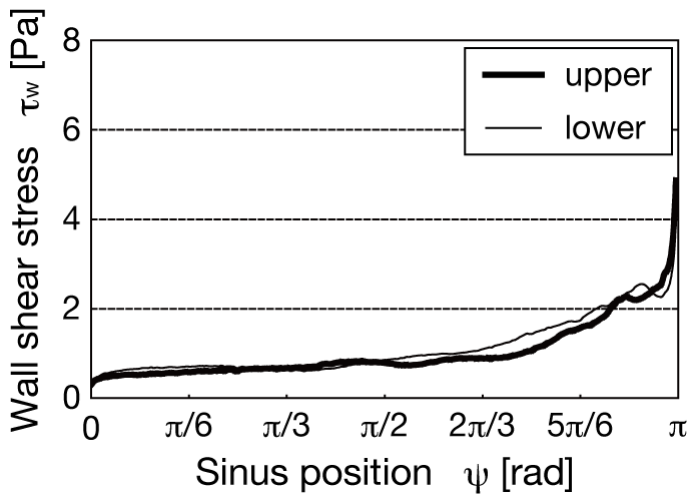

Figure 5: Time-averaged WSS distribution on the wall of sinus of Valsalva. The sinus position $\psi=0$ and $\pi$ [rad] correspond to proximal and distal edges of the sinus of Valsalva, respectively.

In this paper, we apply the virtual flux method to the $2 \mathrm{D}$ aortic valves, and reproduce the blood flow fields around the aortic valves and the sinus of Valsalva by RLBM with LES model. We found that the WSS on the LV-facing surface is larger than that on the aortic-facing surface in cardiac cycle, and the WSS distribution on the wall of sinus of Valsalva is strongly affected by vortices within the sinus.

\section{References}

[1] Fung, Y.C., Biomechanics circulation, $2^{\text {nd }}$ Edition, Springer-Verlag: New York, Berlin, Heidelberg, pp. 42-48, 1997.

[2] Bellhouse, B.J. and Talbot, L., The fluid mechanics of the aortic valve. Journal of Fluid Mechanics, 35, pp. 721-735, 1969.

[3] Van Steenhoven, A.A. and van Dongen, M.E.H., Model studies of the closing behavior of the aortic valve. Journal of Fluid Mechanics, 90, pp. 21-32, 1979.

[4] Fukui, T. and Morinishi, K., Aortic valve oscillation due to vortices in the sinus of Valsalva by virtual flux method. Proceedings of the $8^{\text {th }} \mathrm{KSME-}$ JSME Thermal and Fluids Engineering Conference, Incheon, pp. 1-4, 2012.

[5] Fukui, T. and Morinishi, K., Numerical simulation of blood flows in the aorta with aortic valves by virtual flux method. Proceedings of the European Congress on Computational Methods in Applied Sciences and Engineering, eds. J. Eberhardsteiner et al., Austria, pp. 1-10, 2012.

[6] Kruger, T., Varnik, F. and Raabe, D., Shear stress in lattice Boltzmann simulations. Physical Review E, 79(046704), pp. 1-14, 2009.

[7] Yu, D., Mei, R., Luo, L.S. and Shyy, W., Viscous flow computations with the method of lattice Boltzmann equation. Progress in Aerospace Science, 39, pp. 329-367, 2003. 
[8] Zhang, J., Yan, G. and Shi, X., Lattice Boltzmann model for wave propagation. Physical Review E, 80(026706), pp. 1-13, 2009.

[9] Verschaeve, J.C.G., Analysis of the lattice Boltzmann Bhatnagar-GrossKrook no-slip boundary condition: Ways to improve accuracy and stability. Physical Review E, 80(036703), pp. 1-23, 2009.

[10] Boyd, J., Buick, J., Cosgrove, J.A. and Stansell, P., Application of the lattice Boltzmann model to simulated stenosis growth in a two-dimensional carotid artery. Physics in Medicine and Biology, 50, pp. 4783-4796, 2005.

[11] Tamagawa, M., Kaneda, H., Hiramoto, M. and Nagahama, S., Simulation of thrombus formation in shear flows using lattice Boltzmann method. Artificial Organs, 33(8), pp. 604-610, 2009.

[12] Axner, L., Hoekstra, A.G., Jeays, A., Lawford, P., Hose, R. and Sloot, P.M.A., Simulations of time harmonic blood flow in the Mesenteric artery: comparing finite element and lattice Boltzmann methods. Biomedical Engineering Online, 8(23), pp. 1-8, 2009.

[13] Izham, M., Fukui, T. and Morinishi, K., Application of regularized lattice Boltzmann method for incompressible flow simulation at high Reynolds number and flow with curved boundary. Journal of Fluid Science and Technology, 6(6), pp. 812-821, 2011.

[14] Latt, J. and Chopard, B., Lattice Boltzmann method with regularized precollision distribution functions. Mathematics and Computers in Simulation, 72, pp. 165-168, 2006.

[15] Tanno, I., Morinishi, K., Matsuno, K. and Nishida, H., Validation of virtual flux method for forced convection flow. JSME International Journal, B49(4), pp. 1141-1148, 2006.

[16] Bhatnagar, P.L., Gross, E.P. and Krook, M., A model for collision processes in gases: I. Small amplitude processes in changed and neutral one-component system. Physical Review, 94, pp. 511-525, 1954.

[17] Sterling, J.D. and Chen, S., Stability analysis of lattice Boltzmann methods. Journal of Computational Physics, 123, pp. 196-206, 1996.

[18] Quian, Y.H., d'Humieres, D. and Lallemand, P., Lattice BGK models for Navier-Stokes equation. Europhysics Letters, 17, pp. 479-484, 1992.

[19] Mei, R., Yu, D. and Shyy, W., Force evaluation in the lattice Boltzmann method involving curved geometry. Physical Review E, 65(041203), pp. 114, 2002.

[20] Yu, H., Girimaji, S.S. and Luo L.S., DNS and LES of decaying isotropic turbulence with and without frame rotation using lattice Boltzmann method. Journal of Computational Physics, 209, pp. 599-616 2005. 